\title{
Essentials im atopischen Ekzem-Management
}

\section{Essentials in the Atopic Eczema Management}
Autor
D. Abeck

\section{Bibliografie}

DOI $10.1055 / \mathrm{s}-2007-966326$

Akt Dermatol 2007; 33:

135-136 @ Georg Thieme

Verlag KG Stuttgart · New York ISSN 0340-2541

Korrespondenzadresse

Prof. Dr. med. Dietrich Abeck Bergmannstraße 7 80339 München professorabeck@dermatologiekompetenz.de

\section{Zusammenfassung \\ $\nabla$}

Das insbesondere im Kindesalter sehr häufige atopische Ekzem benötigt eine stadiengerechte Ekzembehandlung unter besonderer Berücksichtigung der Basistherapie. Für die antientzündliche läsionale Behandlung stellen topische Ste-

Das atopische Ekzem ist die häufigste chronische Hauterkrankung im Kindesalter. Für viele Eltern bricht mit der Diagnosestellung „Ihr Kind hat eine Neurodermitis“ die Welt zusammen. Hierfür besteht jedoch in den meisten Fällen überhaupt kein Grund, da die Erkrankung in über $80 \%$ der Fälle leicht verläuft. Die schweren Erkrankungsfälle sind die Ausnahme. Somit kommt dem Erstgespräch mit den besorgten Eltern auch die Bedeutung zu, eine Einschätzung über die Schwere des Ekzems und seiner Prognose zu geben, wobei für die Einordnung die in $\bullet$ Tab. 1 genannten klinischen Gesichtspunkte herangezogen werden können. Zusätzlich ist der Nachweis von IgE-Sensibilisierungen ein weiterer ungünstiger Parameter [7].

\section{Stadiengerechte Behandlung und Ver- meidung von Provokationsfaktoren bilden die zentralen Bestandteile des Managements \\ $\nabla$}

Die sich am klinischen Bild orientierende Behandlung des Ekzems bzw. der trockenen Haut gehört zum festen Bestandteil jedes therapeutischen Ansatzes wie auch die konsequente Aufdeckung und Vermeidung möglicher irritativer oder allergischer Provokationsfaktoren.

\section{Basistherapie}

Obwohl bis heute nicht gezeigt werden konnte, dass durch den Einsatz von Basisexterna das Auf- roide der 4. Generation sowie topische Calcineurin-Inhibitoren die Substanzen der Wahl dar. Das konsequente Meiden irritativer Faktoren sowie nach entsprechender Testung allergischer Provokationsfaktoren flankiert die therapeutischen Maßnahmen.

treten eines atopischen Ekzems im Sinne einer Primärprävention verhindert werden kann [10], ist ihr Stellenwert im Rahmen der Sekundärprävention gut belegt. Dies zeigen auch die aktuellen Daten der über 2000 Patienten umfassenden ATOPA-Studie. Eine konsequente Basistherapie, wobei eine Creme mit N-Palmitoylethanolamin (Physiogel $^{\circledR}$ A.I. Creme) zum Einsatz gelangte, zeigte signifikante Verbesserung hinsichtlich des klinischen Bildes als auch der subjektiven Parameter Juckreiz und Schlafverlust [2].

\section{Topische antiinflammatorische Behandlung: Steroide und Topische Calcineurin-Inhibitoren \\ $\nabla$}

Eine Abheilung der Ekzeme ist häufig auch unter einer konsequenten Basistherapie nicht möglich, so dass zusätzlich antientzündlich wirksame Substanzen zum Einsatz gelangen müssen. Neben den topischen Steroiden der 4. Generation, $\mathrm{zu}$ denen Hydrocortisonaceponat (Retef ${ }^{\circledR} \mathrm{AP}$ ), Hydrocortisonbuteprat $\left(\right.$ Pandel $\left.^{\circledR}\right)$, Hydrocortison-17-butyrat (Alfason ${ }^{\circledR}$, Laticort ${ }^{\circledR}$ ), Methylaceponat $\left(\right.$ Advantan $\left.^{\circledR}\right)$, Prednicarbat (Dermatop ${ }^{\circledR}$, Prednitop ${ }^{\circledR}$ ) gehören, stehen die topisch anwendbaren Makrolaktame Pimecrolimus und Tacrolimus als Steroidalternativen zur Verfügung, die über Vorteile [14], jedoch auch Nachteile [1] gegenüber den topischen Steroiden verfügen. In der täglichen Praxis ist auch der gleichzeitige oder alternierende Einsatz der topischen Steroide 
Kriterien für das leichte atopische Ekzem mit günstiger Prognose

Auftreten nach dem 6. Lebensmonat nummuläre Variante

lokalisierte Verläufe mit Ausnahme des Gesichts geringe Ekzeminfiltration geringer Juckreiz
Kriterien für das schwere atopische Ekzem mit schlechter Prognose

Auftreten bereits in den ersten 3 Lebensmonaten Prurigoform

disseminierte oder generalisierte Verläufe

stärkere Ekzeminfiltration

stärkerer Juckreiz und der Calcineurin-Inhibitoren häufig für eine befriedigende Ekzemstabilisierung notwendig.

Leider stehen weitere vergleichbar wirksame Alternativen nicht zur Verfügung. Dies gilt für Gerbstoff-, Phytopharmaka- oder für Schieferöl-haltige Externa. Neben einer geringen Wirksamkeit besteht für Bufexamac zusätzlich das Risiko einer Auslösung schwerer, auch generalisiert verlaufender allergischer Reaktionen. Eine aktuelle Untersuchung zur Häufigkeit von Typ IV-Sensibilisierungen zeigte keinen Unterschied zwischen Kindern mit atopischem Ekzem und einer nicht-atopischen Kontrollgruppe mit einer Ausnahme: bei den Kindern mit atopischem Ekzem bestand eine dreimal häufigere Kontaktsensibilisierung gegenüber Bufexamac [6].

\section{Gegen Staphylococcus aureus-gerichtete Therapiemaßnahmen}

Die Hautflora von Patienten mit atopischem Ekzem unterscheidet sich von der von Patienten ohne atopisches Ekzem hinsichlich der Kolonisation mit dem grampositiven Bakterium Staphylococcus aureus. Der Erreger ist in der Lage, Entzündungsreaktionen über Toxine und Superantigene auszulösen und zu unterhalten [3]. Unlängst konnte auch ein verstärkender Effekt von Superantigenen des Erregers auf durch Hausstaubmilben-induzierte Patchtestungen gezeigt werden [8].

Die durch den Einsatz von Antiseptika wie z.B. Triclosan reduzierte Besiedelung der Haut mit S. aureus korreliert mit einer Ekzemverbesserung. Auch der kombinierte Einsatz von Triclosan und Chlorhexidin in einer kommerziell erhältlichen Zubereitung unter Verwendung von für den kosmetischen Bereich zugelassenen Konzentration von 0,3\% Triclosan und 0,34\% Chlorhexidin (Lipoderm Lotion ${ }^{\circledR}$ ) resultiert in einer Ekzemverbesserung [13]. Neben dem Einsatz von Antiseptika haben sich auch funktionelle Textilien mit antimikrobiellen Zusätzen in Form von mit einer quarternären Ammoniumverbindung beschichteten Seide [9] oder einer silberbeschichteten Mikrofaser [5] bewährt.

\section{Hausstaubmilben als wichtiger allergischer Provokationsfakor}

Insbesondere bei Auftreten von Ekzemen in frei getragenen Arealen wie Gesicht, Hals und Dekolleté ist an Hausstaubmilben als Provokationsfaktor zu denken und eine entsprechende $\mathrm{Ab}$ klärung sinnvoll. Neben Hausstaubmilben-reduzierenden Maßnahmen wie dem Einsatz von Hausstaubmilben undurchlässigen aber atmungsaktiven Matratzen-, Bettdecken- und Kopfkissenüberzügen („Encasings“) kann auch eine spezifische Immuntherapie eine geeignete Therapiemaßnahme darstellen [11].

\section{Hitliste der irritativen Provokationsfaktoren}

Schwitzen, insbesondere bei körperlicher Anstrengung, unsachgemäße Kleidung wie z.B. Wolle und heißes Wetter sind aufgrund einer aktuellen Untersuchung bei Schulkindern die wichtigsten Provokationsfaktoren des atopischen Ekzems [12]. Ein 4-wöchiger Aufenthalt in einem milden, UV-reichen Klima führt zu einer deutlichen Ekzemverbesserung mit einer auch noch nach drei Monaten nach Rückkehr nachweisbaren Stabilisierung, was eine Untersuchung bei 4- bis 13-jährigen Kindern aus Norwegen mit Aufenthalt auf Gran Canaria zeigte [4].

\section{Abstract}

\section{Essentials in the Atopic Eczema Management $\nabla$}

Atopic eczema is a frequent chronic disease manifestating especially in early childhood. A disease-related anti-inflammatory treatment in combination with adjuvant basic therapy is mandatory. Topical $4^{\text {th }}$ generation steroids and topical calcineurin-inhibitors are the anti-inflammatory substances of choice. The consequent avoidance of irritatative and individual allergic provocation factors based on appropiate testing support active dermatological treatment.

\section{Literatur}

1 Abeck D. Topische Calcineurin-Inhibitoren bei atopischem Ekzem Contra. DMW 2006; 131: 1615

2 www.atopa.de/atopa_studie.htm

3 Baker BS. The role of microorganisms in atopic dermatitis. Clin Exp Dermatol 2006; 144: 1 -9

4 Byremo S, Rod G, Carlsen $\mathrm{KH}$. Effect of climate change in children with atopic eczema. Allergy 2006; 61: $1403-1410$

5 Gauger A, Fischer S, Mempel M, Schaefer T, Foelster-Holst R, Abeck D, Ring $J$. Efficacy and functionality of silver-coated textiles in patients with atopic dermatitis. J Eur Acad Dermatol Venereol 2006; 20: 534-541

6 Heine G, Schnuch A, Uter W, Worm M. Type IV-sensitization profile of individuals with atopic eczema: results from the Information Network of Departements of Dermatology (IVDK) and the German Contact Dermatitis Research Group (DKG). Allergy 2006; 61: 611-616

7 Illi S, von Mutius E, Lau S, Nickel R, Gruber C, Niggemann B, Wahn U. The natural course of atopic dermatitis from birth to age 7 years and the association with asthma. J Allergy Clin Immunol 2004; 113: 925 - 931

8 Langer K, Breuer K, Kapp A, Werfel T. Staphylococcus aureus-derived enterotoxins enhance house dust mite-induced patch test reactions in atopic dermatitis. Exp Dermatol 2007; 16: 124-129

9 Senti G, Steinmann LS, Fischer B, Kurmann R, Storni T, Johansen P, Schmid-Grendelmeier P, Wüthrich B, Kundig TM. Antimicrobial silk clothing in the treatment of atopic dermatitis proves comparable to topical steroid treatment. Dermatology 2006; 213: 228-233

10 Simpson EL. Atopic dermatitis prevention. Dermatol Ther 2006; 19: $108-117$

11 Werfel T, Bruer K, Rueff F, Przybilla B, Worm M, Grewe M, Ruzicka T, Brehler R, Wolf H, Schnitker J, Kapp A. Usefulness of specific immunotherapy in patients with atopic dermatitis and allergic sensitisation to house dust mites: a multi-centre, randomised, dose-response study. Allergy 2006; 61: $202-205$

12 Williams JR, Burr ML, Williams HC. Factors influencing atopic dermatitis - a questionnaire survey of schoolchildren's perceptions. Br J Dermatol 2004; 150: 1154-1161

13 Wohlrab J, Jost G, Abeck D. Antiseptic efficacy of a low-dosed topical triclosan/chlorhexidine combination therapy in atopic dermatitis. Skin Pharmacol Physiol 2006; 20: 71 - 76

14 Wollenberg $A$. Topische Calcineurin-Inhibitoren bei atopischem Ekzem - Pro. DMW 2006; 131: 1614 